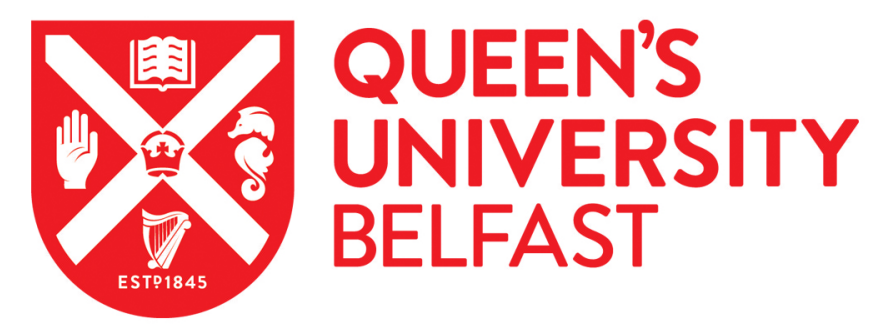

\title{
A Frequency-Dependent Equivalence Between Groove Gap Waveguide and Rectangular Waveguide
}

Berenguer, A., Fusco, V., \& Baqueo-Escudero, M. (2016). A Frequency-Dependent Equivalence Between Groove Gap Waveguide and Rectangular Waveguide. In 2016 IEEE International Symposium on Antennas and Propagation (APSURSI) Institute of Electrical and Electronics Engineers Inc.. https://doi.org/10.1109/APS.2016.7696255

Published in:

2016 IEEE International Symposium on Antennas and Propagation (APSURSI)

Document Version:

Peer reviewed version

Queen's University Belfast - Research Portal:

Link to publication record in Queen's University Belfast Research Portal

Publisher rights

(c) 2016 IEEE. Personal use of this material is permitted. Permission from IEEE must be obtained for all other uses, in any current or future media, including reprinting/republishing this material for advertising or promotional purposes, creating new collective works, for resale or redistribution to servers or lists, or reuse of any copyrighted component of this work in other works.

\section{General rights}

Copyright for the publications made accessible via the Queen's University Belfast Research Portal is retained by the author(s) and / or other copyright owners and it is a condition of accessing these publications that users recognise and abide by the legal requirements associated with these rights.

Take down policy

The Research Portal is Queen's institutional repository that provides access to Queen's research output. Every effort has been made to ensure that content in the Research Portal does not infringe any person's rights, or applicable UK laws. If you discover content in the Research Portal that you believe breaches copyright or violates any law, please contact openaccess@qub.ac.uk. 


\section{A Frequency-Dependent Equivalence Between Groove Gap Waveguide and Rectangular Waveguide}

\author{
Antonio Berenguer, Vincent Fusco ${ }^{+}$, Mariano Baquero- \\ Escudero \\ Instituto de Telecomunicaciones y Aplicaciones Multimedia \\ Universitat Politècnica de València \\ Valencia (46022), Spain
}
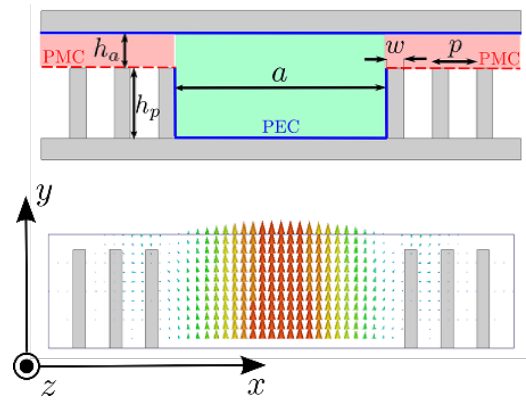

Fig. 2. GGW fundamental mode

anbever@iteam.upv.es

\begin{abstract}
Recently gap waveguides have been shown as a potential alternative to conventional waveguides in the millimeterwave band. Groove Gap Waveguide (GGW) has until now been studied though direct correspondence with rectangular waveguide with the same propagation channel dimensions. However there have been observed differences in the above cutoff propagation characteristics between these waveguide types. Furthermore, the behaviour of GGW below cutoff remains unknown. This work presents a discussion of below and above cutoff propagation characteristics, and introduces a simple model that explains observed GGW behavior and establishes its propagation characteristics.
\end{abstract}

Index Terms-groove gap waveguide, transmission lines, impedance, evanescent propagation.

\section{INTRODUCTION}

The millimeter-wave band [1]-[4] continues to attract the interest of the research community as new applications consistently demand the development of suitable components. At millimeter wave bands, dielectric materials can have high losses [5]-[6] and coupling to substrate modes [7]-[8] can be problematical. Recently, in an attempt to solve these problems, Gap Waveguide (GW) was proposed [10]-[11]. GW is based on the use of a periodic structure, usually realized by square metal pins, Fig. 1. The pin lattice introduces a high impedance condition at the plane above the pins. Thus, by placing a metal plate at a distance $h_{a}=\lambda / 4$ from the top of the pins, over a certain frequency range defined by the periodic lattice parameters, no wave can propagate in this region. Groove Gap Waveguide (GGW) can take two versions, vertical polarization (VP), Fig. 1(a), and horizontal polarization (HP), Fig. 1(b). Both versions act in a similar manner to a rectangular waveguide, propagating a quasi- TE mode.

GGWs have shown their potential advantage versus conventional waveguide through prototypes, including couplers, filters and antennas [12]-[15]. However, at this moment, although the behavior of the periodic lattice is characterized [16], it seems that more effort is necessary in the development of simple models that explain better GGW propagation characteristics especially close to, and below cut-off.

The difficulty in characterizing gap waveguide arises from the presence of the periodic pin structure, which leads to a

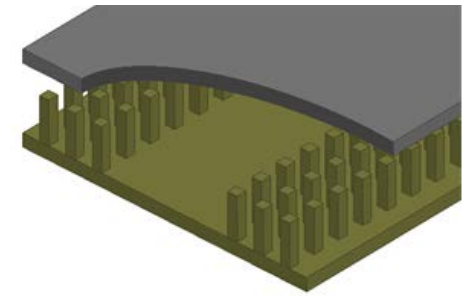

a) GGW-VP

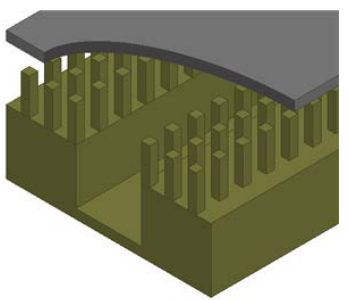

b) GGW-HP
Fig. 1. Gap waveguides.

waveguide which is not homogeneous in the propagation direction and has many design parameters. Homogenization of the structure based on metamaterial analogies has led to analytical models [17]-[19], however unlike metamaterials, the periodic structure in GGW can be comparable with the operating wavelength, thus calling into question their general applicability, [17]-[21].

In particular the propagation characteristics of GGW have not been analyzed in great detail. Usually it is assumed that GGW behaves like a rectangular waveguide with the same propagation channel dimensions [20]. However, important differences between GGW and rectangular waveguide will in this paper be shown to exist. GGW behavior near to, or below, cutoff has to date, to the authors knowledge, not been reported. Below cutoff aspects are important since it is known that waveguide evanescent components can be compact and be made to exhibit spurious free responses [22]-[25].

The main aim of this paper is to address the aforementioned questions. Moreover, it presents a simple model which shows agreement with simulated results and provides a simple explanation of how GGW operates. It is also is useful as a tool for extracting the influence of the different waveguide parameters in the dispersion diagram though fast parametric analysis, thus avoiding the need for very time consuming fullwave EM simulation. The remainder of this paper will focus on GGW-VP' ${ }^{1}$, which is the option, to date, chosen to implement high quality resonators [26] and low insertion loss filters [13], [27]. 
II. THE PAPER IS ORGANIZED AS FOLLOWS. IN SECTION II THE GROOVE GAP WAVEGUIDE IS PRESENTED, AND ITS PROPAGATION CHARACTERISTICS ARE STUDIED BOTH BELOW AND ABOVE CUTOFF. THE OBSERVED DIFFERENCES BETWEEN THIS WAVEGUIDE AND CLASSICAL RECTANGULAR WAVEGUIDE ARE DISCUSSED. IN SECTION III A SIMPLE PROPAGATION MODEL IS PROPOSED AND THE RESULTS OBTAINED SHOWN. IN SECTION

IV A PARAMETRIC STUDY OF THE PERIODIC STRUCTURE IS CARRIED OUT. FINALLY, CONCLUSIONS AND OVERALL REMARKS ARE GIVEN. GROOVE GAP WAVEGUIDE

The GGW was first proposed in [10]. Fig. 2 shows the

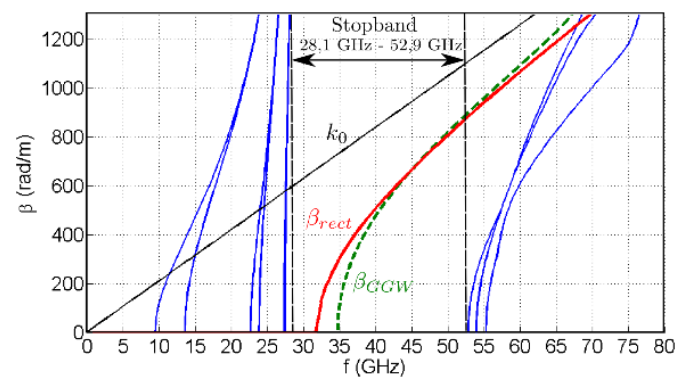

Fig. 2. GGW dispersion diagram

transverse view of this type of waveguide, its main geometrical parameters and fundamental, propagating mode. The periodic structure inhibits propagation in lateral directions and imposes a propagation mode similar to the $\mathrm{TE}_{01}$ mode of standard rectangular waveguide.

Although the lateral periodic structures should in theory be of infinite extent, in practice they can be significantly truncated without significant loss of performance. Three rows of pins have been shown as sufficient to achieve the desired effect of forbidden propagation [13], [26].

\section{A. Operation above cutoff}

To analyze the dispersion diagram of the structure the dimensions used in [13] are taken for reference. These dimensions are $h_{p}=2.4 \mu \mathrm{m}, w=0.3 \mu \mathrm{m}, p=0.9 \mu \mathrm{m}, h_{a}=0.375 \mu \mathrm{m}$ and $a=4.7 \mu \mathrm{m}$. All simulations are carried out using CST $^{\circledR}$ [28]. Fig. 3 shows the propagation constant of the first modes for this structure. The black curve is the propagation constant of a plane wave, blue curves correspond to unwanted modes and the green dashed curve is the desired mode. The propagation constant of a rectangular waveguide with same dimensions as the propagation channel of the GGW is displayed for comparison. It can be seen that the band [28.1 GHz $-52.9 \mathrm{GHz}$ ] represents the stopband of the periodic structure, so that here only the desired mode propagates in the waveguide.

In previous works, [13], [20], [27], it has been assumed that the analogue of a GGW is a rectangular waveguide having the same propagation channel dimensions. However, when comparing the curves in Fig. 3 of the GGW with those of the rectangular waveguide it can be said that in the upper half of the stop band both curves are similar, but that this is no longer true at near cutoff. In fact, both waveguides present a different cutoff

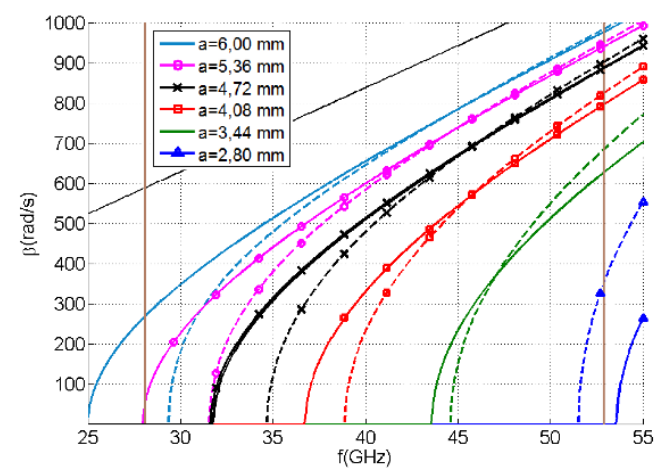

Fig. 4. Propagation constant of discussed GGW and rectangular waveguide for different values of $a$.

frequency, and even for frequencies where they are similar a greater dispersive behavior is observed for the GGW structure.

To further characterize the propagation properties of GGW, the width of the propagation channel $a$ is parameterized. To cover the possible cases of having cutoff going from near the minimum frequency of the stopband to near the maximum frequency of the stopband, six values of $a$ equally distributed between $a=2.8 \mu \mathrm{m}$ and $a=6 \mu \mathrm{m}$ (both included) are considered. The standard waveguide configuration is represented by the curve $a=4.72 \mu \mathrm{m}[13]$.

The results of this parameterization are shown in the Fig. 4. Here, in most cases, the GGW presents a higher cutoff frequency than its rectangular waveguide counterpart, especially as $a$ increases. This means that GGW is effectively smaller in propagating aperture than the equivalent rectangular waveguide. The contrary could be expected since in GGW the fields are not strictly transmitted in the channel, but spread evanescently through the lateral pin regions. As $a$ decreases this difference becomes smaller and, if $a$ is small enough ( $a=2.8 \mu \mathrm{m}$ the GGW has lower cutoff frequency than the rectangular waveguide, and becomes effectively larger than the rectangular waveguide.

In general, simulation reveals GGW to exhibit a more dispersive behavior when compared with rectangular waveguide. For the cases of larger $a$ the GGW curve grows faster with frequency and reaches the rectangular waveguide curve, and, although it surpasses it, both curves are quite similar from that point. For the cases of smaller $a$, this difference is greater and both curves diverge having only a very narrow band of coincidence, or even no coincidence e.g. at $a=2.8 \mu \mathrm{m}$ where a very dispersive curve is observed.

From the above study the standard assumption of equivalence between rectangular waveguide and GGW is valid only in a certain frequency bands determined by specific range values of $a$.

\section{B. Operation below cutoff}

In a below cutoff rectangular waveguide the lateral conditions are electric walls and evanescent energy is delivered along the axial direction only. In the GGW, the condition of forbidden propagation into the pins regions permits also exponential decay as a lateral condition [17]. Consider the following example; the electric field is simulated for the case $a=4.72 \mathrm{~mm}$, which implies a cutoff of $f_{c}=34.68 \mathrm{GHz}$ in 
rectangular waveguide. Since the stopband of the GGW structure starts at $f=28.1 \mathrm{GHz}$, three frequencies are analyzed, $f=28 \mathrm{GHz}$ (outside the stopband, mode below cutoff), $f=29 \mathrm{GHz}$ (inside the stopband, mode below cutoff), and $f=40 \mathrm{GHz}$ (inside the stopband, mode under usual operation). The results of this comparison are shown in the Fig. 5. As can be seen, outside the stopband, the field spread into the pin structure and attenuation is increased while when inside the stopband energy is delivered along the axial direction in a similar manner to the rectangular waveguide.

It is observed that the field spreads more in the lateral directions when the mode is below cutoff. This can be seen in Fig. 6 where the $E_{y}$ component as a function of $x$ on a transversal plane $z=z_{0}$ for different heights is plotted. The frequencies considered are $f=29 \mathrm{GHz}$ (far below cutoff), $f=34 \mathrm{GHz}$ (near below cutoff), $f=40 \mathrm{GHz}$ (normal operation), and $f=52 \mathrm{GHz}$ (far above cutoff). It is seen that the field is better confined in the propagation channel as frequency increases. Near to cutoff axial attenuation is lower suggesting that the lateral conditions are presenting a higher attenuation path. Above cutoff lower interaction with the GGW lateral walls occur. These results suggest that GGW can operate below cutoff in an analogous manner to rectangular waveguide. However the differences between both waveguide types are accentuated.

We now study the dispersion diagram of the structure under cutoff operation. Above cutoff the calculation of propagation constant given in [29] is sufficient. Below cutoff we simulate the entire structure, Fig. 5(a), and evaluate,

$$
\alpha\left(\frac{N p}{m}\right)=\frac{\ln \frac{E_{y}\left(z_{1}\right)}{E_{y}\left(z_{z}\right)}}{z_{1}-z_{2}}
$$

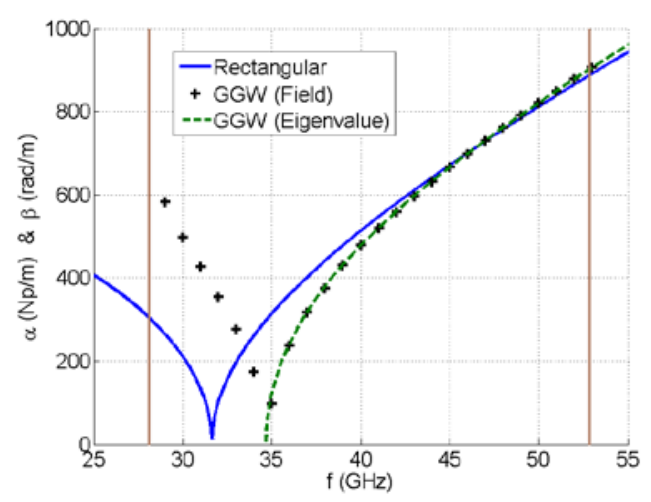

Fig. 7. Propagation and attenuation constant for a rectangular waveguide and a GGW of $a=4,72 \mu \mathrm{m}$

here $z_{1}>z_{2}$.

The results of this study are shown in Fig. 7, which displays the attenuation and propagation constants of a rectangular waveguide (analytical) and a GGW (eigenvalue computation, and field computation of the full structure) for the case of $a=4.72$ $\mu \mathrm{m}$. a) GGW-VP

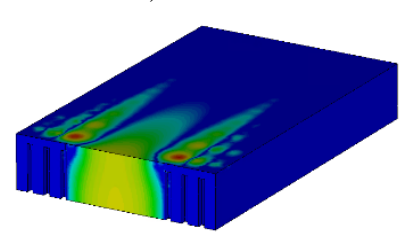

c) GGW-VP

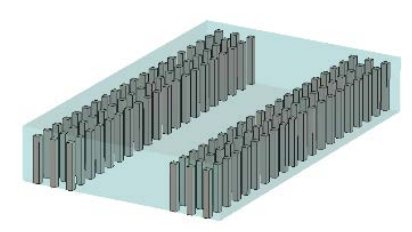

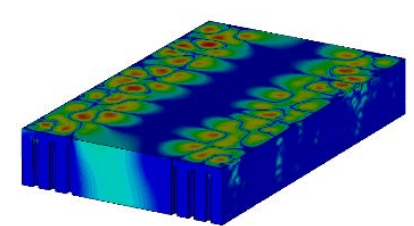

b) GGW-HP

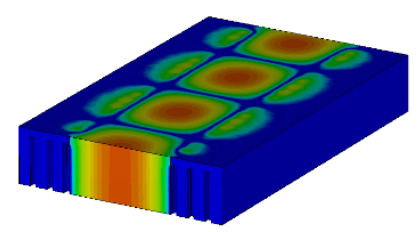

d) GGW-HP
Fig. 5. $E_{y}$ field inside a GGW with a=4,72 $\mathrm{mm}$ for different classes of propagation.

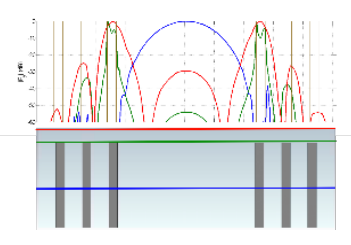

a) $f=29 \mathrm{GHz}$

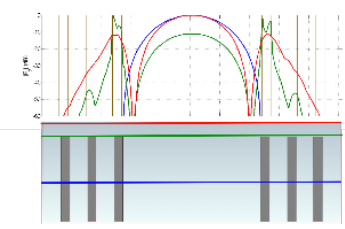

c) $\mathrm{f}=40 \mathrm{GHz}$

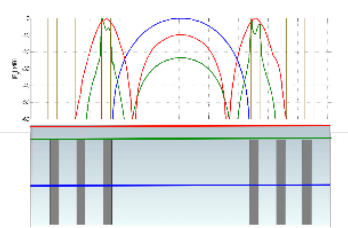

b) $\mathrm{f}=34 \mathrm{GHz}$

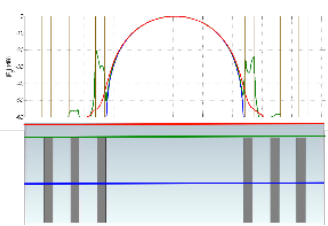

d) $\mathrm{f}=52 \mathrm{GHz}$
Fig. 6. $E_{y}(x) \mathrm{GGW}$ with $\mathrm{a}=4,72 \mu \mathrm{m}$ on a transversal plane at different heights $y_{i}$. Blue line is $y=h_{p} / 2$, green line is $y=h_{p}$, and red line is $y=h_{p}+h_{a}$.

Fig.7 indicates that the difference between the curves for both waveguide types continue increasing when the frequency goes below cutoff. Further, as the frequency decreases, the attenuation in the GGW grows faster than in the rectangular waveguide. Furthermore GGW exhibits growth as the stopband limit approaches instead of the expected slope decrease. Similar results have been observed for other cases of $a$, indicating that rectangular waveguide and GGW behave noticeably different below cutoff.

\section{PROPOSED MODEL}

From the previous study it is clear that assuming that for identical $a$ GGW behaves equivalently to a rectangular waveguide implies error unless $f_{c}$ is near to the low stopband limit and that frequency of operation is far enough from cutoff. Moreover modelling this behavior is not possible by scaling the rectangular waveguide by a constant factor since a frequency dependent behaviour is observed. The shape of the obtained curves indicates that a mechanism is occurring within the structure which involves reactance being introduced by the periodic lattice. 


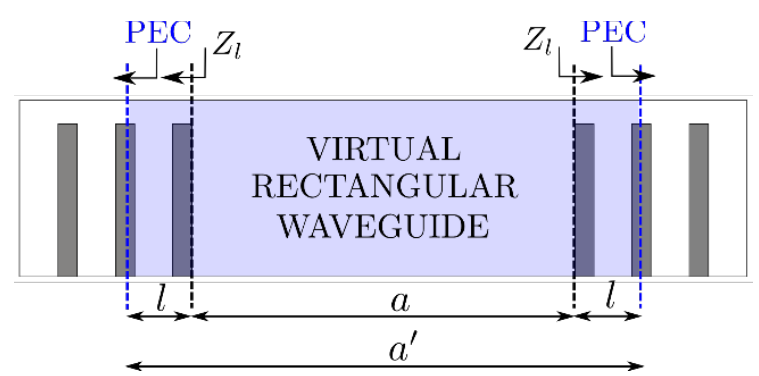

Fig. 8. Schematic of the employed method

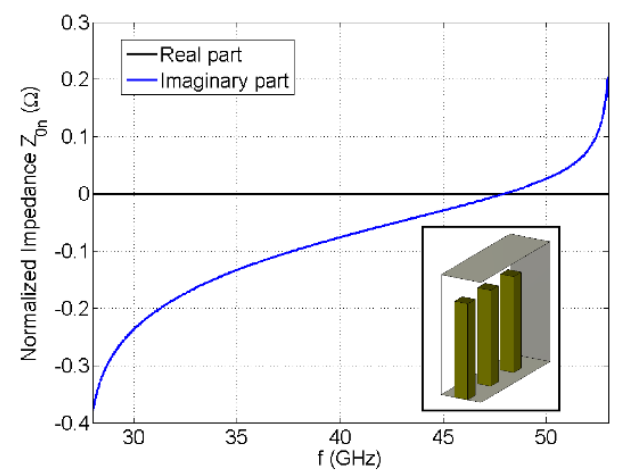

Fig. 9. Lateral impedance viewed at the first pin row plane.

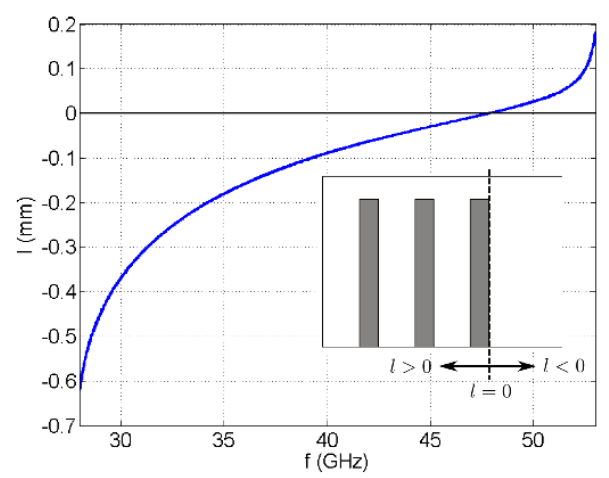

Fig. 10. Distance $l$ at which a PEC wall would produce the same impedance as the periodic structure.

\section{A. Employed Model}

Consider GGW, but now with regard to propagation in the lateral direction $x$. What is of interest is the impedance $Z_{l}$ seen looking into the first row of pins, Fig. 8, where $Z_{l}$ is the impedance of the waves incident on the sidewall. At cutoff $\beta=$ 0 so that propagation is completely in the transversal direction. The port is placed on the first pin with the rest of the arrangement terminated as a perfect electric conductor (PEC). Hence referred to this port the normalized impedance of the structure is

$$
Z_{\text {ln }}=\frac{1+S_{11}}{1-S_{11}}
$$

To obtain the required $S_{11}$, the structure is simulated using CST. For canonical shapes further reductions in computing time are possible, [30]-[31]. With the model in Fig.8 only a small part of a periodic cell is discretized and solved for its scattering parameters. This results in a significantly faster solution process than by direct eigenvalue solution [36]. Moreover, once the structure is solved, the results obtained for the lateral impedance are valid for any $a$ while other approaches require a new simulation for every $a$. The resulting normalized impedance by this approach is shown in the Fig. 9. We can see that the periodic structure presents a reactance that exhibits capacitive behaviour at the beginning of the stopband changing to inductive behavior at the end of the stopband crossing zero at $f=47.94 \mathrm{GHz}$.

Let now consider the normalized input impedance of transmission line of characteristic impedance $Z_{0}$ loaded with an impedance $Z_{L}$ which for $Z_{L}=0$, i.e., PEC terminated becomes

$$
Z_{\text {in }}=j \tan (\beta l)
$$

hence

$$
l=-\frac{1}{\beta} \tan ^{-1}\left(j Z_{\text {in }}\right)
$$

Thus the GGW is equivalent to a rectangular waveguide having lateral walls positioned at a distance which depends on $Z_{l n}$. We have therefore a virtual rectangular waveguide with $a^{\prime}=a+2 l$ (see Fig. 8).

Since for small arguments the function $\tan ^{-1}(x)$ is almost linear, the behaviour of both $Z_{l n}$ and $l$ is quite similar. Depending on the sign of $l$, the GGW will be equivalent to a smaller $(l<0)$ or a larger $(l>0)$ rectangular waveguide, as shown in the inset in Fig.10.

\section{B. Results}

The cutoff frequency of the fundamental mode in a rectangular waveguide is:

$$
f_{c}=\frac{c}{2 a}
$$

Since in the proposed model for the GGW the equivalent width $a^{\prime}$ depends on the frequency the term $f_{c}$ will also have this dependence.

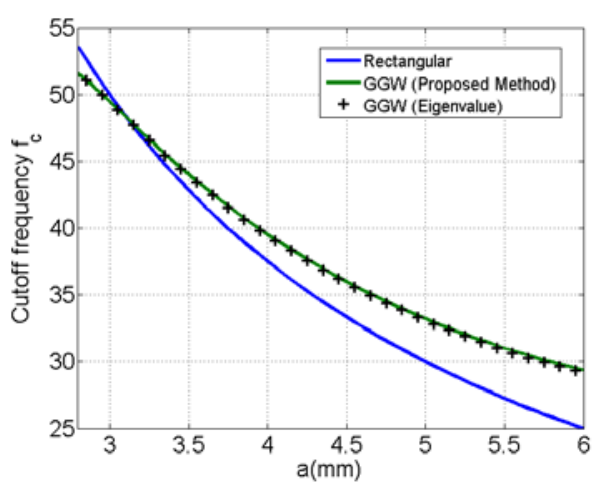




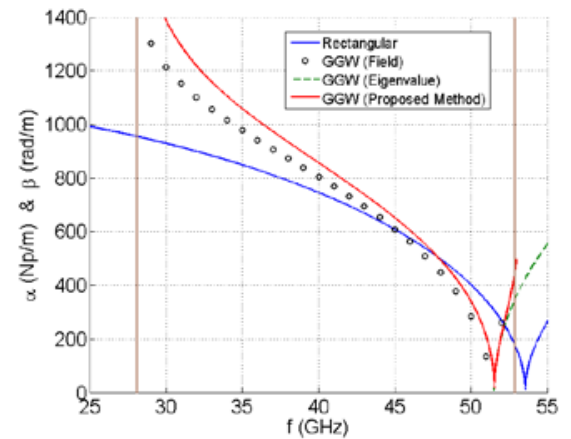

(a) $a=2.80 \mathrm{~mm}$

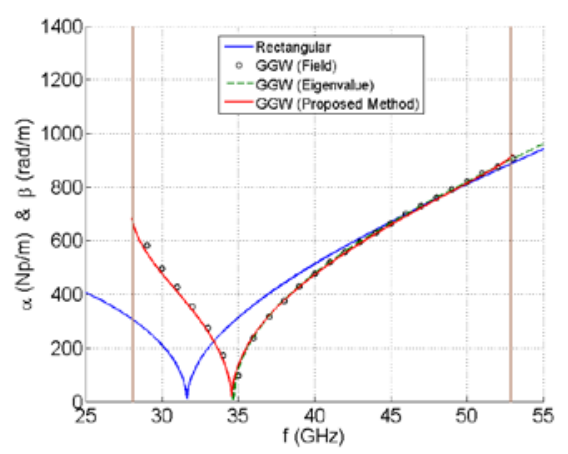

(a) $a=4.72 \mathrm{~mm}$

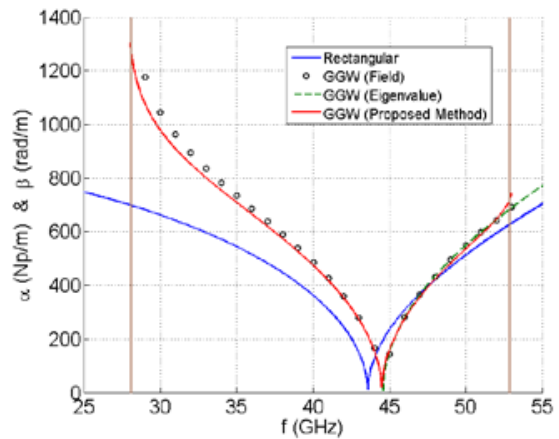

(a) $a=3.44 \mathrm{~mm}$

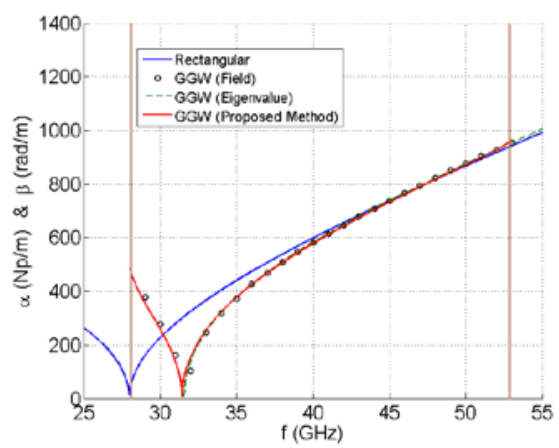

(a) $a=5.36 \mathrm{~mm}$

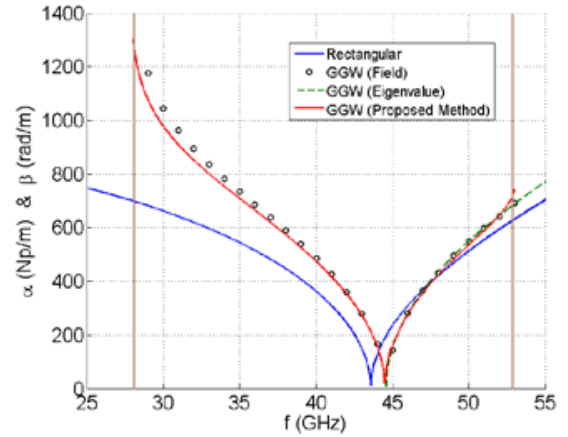

(a) $a=4.08 \mathrm{~mm}$

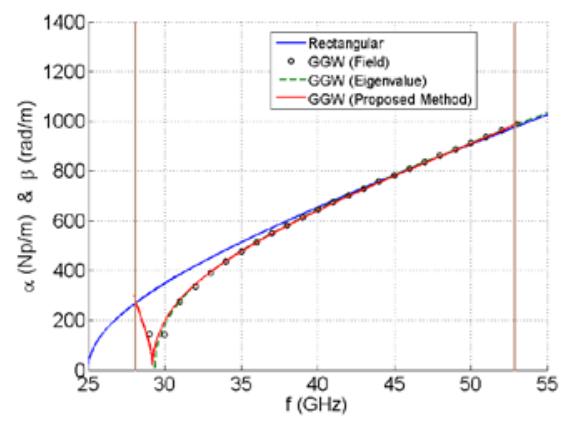

(a) $a=6.00 \mathrm{~mm}$

Fig.12 Simulated propagation and attenuation constants of rectangular waveguide and GGW.

Fig. 11. Lateral impedance viewed at the first pin row planes.

For a given frequency $f_{0}$, one has $a^{\prime}\left(f_{0}\right)$ and by (5), $f_{c}\left(f_{0}\right)$. A zero-finding routine is applied to $y(f)=f_{c}(f)-f$, in order to obtain the cutoff frequency for the GGW. In Fig. 11 the cutoff frequency as a function of $a$ for both rectangular and GGW, comparing eigenvalue solution and proposed method are given. The time spent in the zero-finding routine is negligible.

The propagation or attenuation constant of the GGW can now be obtained through the standard rectangular waveguide formulas:

$$
\begin{array}{ll}
\beta=\sqrt{k^{2}-\left(\frac{\pi}{a^{\prime}}\right)^{2}} \quad & f \geq f_{c} \\
\alpha=\sqrt{\left(\frac{\pi}{a^{\prime}}\right)^{2}-k^{2}} & f<f_{c}
\end{array}
$$

From Fig. 12 it can be seen how the presented method shows good agreement with the full numerically intensive eigenvalue problem solutions and field examination. The proposed method is therefore interesting for component optimization due to its speed and additional physical insight on GGW behavior.

Above cutoff as $a^{\prime}$ grows with frequency (6) implies for GGW that $\beta$ grows with the frequency faster than in the rectangular waveguide case. When the term $k^{2}$ is large compared with $(\pi / a)^{2}$ the variation of $a^{\prime}$ is less significant and the propagation behavior is similar to that of standard rectangular waveguide. This occurs for large $a$ and high frequencies.

Below cutoff as frequency is reduced the term $k^{2}$ becomes small compared with $(\pi / a)^{2}$, thus regarding (7) $\alpha$ exhibits growth with the decrease of $a^{\prime}$ with frequency. This effect explains why the $\alpha$ curve of the GGW does not exhibit a reduction of its slope as occurs with the rectangular waveguide when the frequency decreases. Below cutoff conditions under which the rectangular waveguide and GGW of same propagation channel dimensions behave similarly could not be found. Due to the Foster Reactance Theorem [37] translated though (4) GGW should exhibit greater or equal dispersion than the equivalent rectangular waveguide.

\section{PARAMETERIZATION OF THE PERIODIC STRUCTURE}

Depending on the final application, e.g. [35], [36], it would be desirable to be able to engineer the dispersive behaviour of the GGW. Regarding (6) and (7) the dispersion curves can be controlled if the adequate frequency response of the effective width of the GGW, $a^{\prime}(f)$, can be achieved by properly designing the periodic structure and obtaining the correct $Z_{\text {ln }}(f)$. This section now studies the influence that different periodic structure parameters have on $Z_{l n}(f)$. 

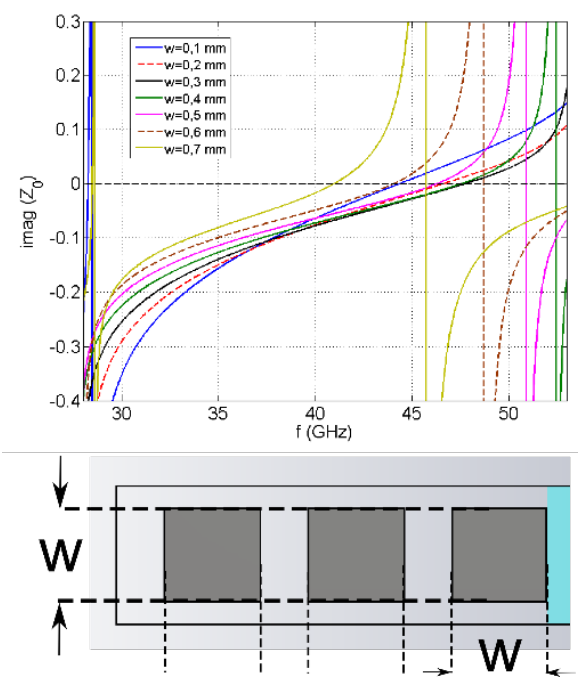

Fig. 13. $Z_{\text {ln }}(f)$ vs. $w$
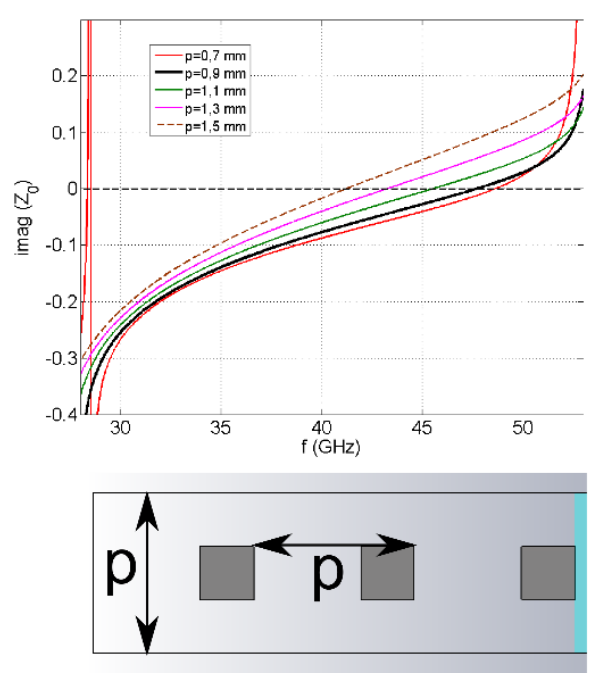

Fig. $14 . Z_{l n}(f)$ vs. $p$

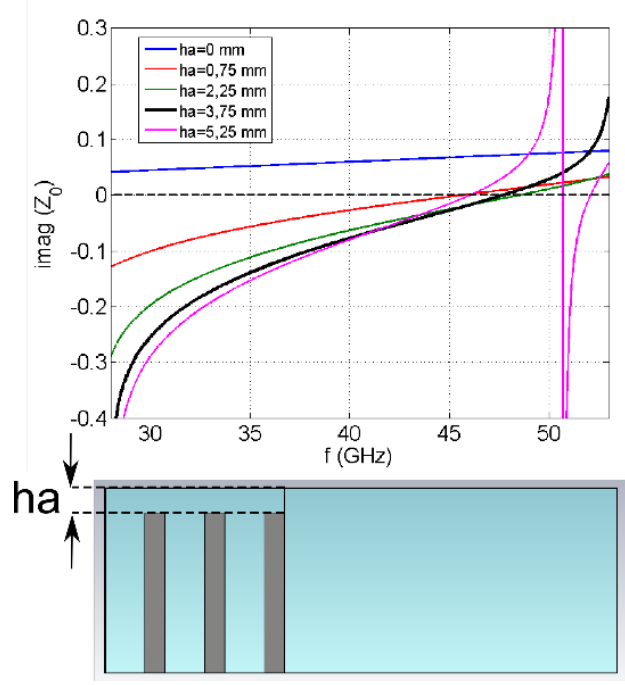

Fig. $15 Z_{l n}(f)$ vs. $h_{a}$

\section{A. Model based parameters}

The main parameters of the periodic structure are given in Fig. 2. The influence of $w$ is shown in the Fig. 13, where $Z_{l n}(f)$ is displayed for several cases of this parameter. In this graph and the following ones, the original value of the parameter correspond to the thickest black curve and the frequency range corresponds to the original stopband.

It is observed that an increase of pin width leads to a reduction of stopband bandwidth hence a more dispersive waveguide. Also as $w$ is increased the capacitances between the pins and between each pin and the top plate increases. This implies a lower negative contribution to the total reactance for a given frequency (larger values of the reactance). On reducing $w$, the stopband slightly increases and moves to higher frequencies. For this case the inductance introduced by the pins increases. This can be observed when $w=0.1 \mathrm{~mm}$ which shows a larger slope in the central zone of the stop band. In Fig. 14 period $p$ is parameterized. Larger periods produce a wider stopband, however, it is observed that those cases exhibit a greater slope in the impedance curve, as well as displacement of the resonance towards the center of the stopband.
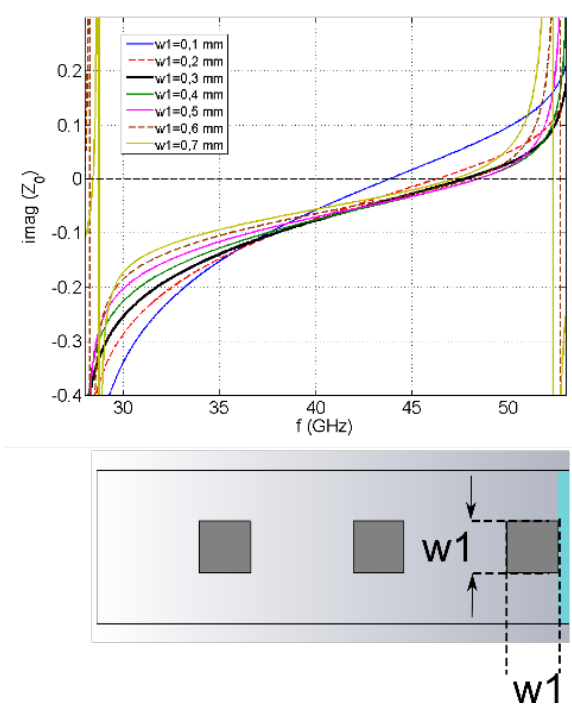

Fig. 16. $Z_{l n}(f)$ vs. $w_{1}$

This effect seems to be caused by the decrement of the capacitances between pins which compensates, to an extent, pin inductance. However, the effect of pin period is less strong than that observed for pin width. It should be noted that very small periods imply that three rows of pins are insufficient to achieve the desired effect of lateral attenuation.

Finally, the distance $h_{a}$ at which is placed the top metal plate is parameterized, Fig. 15. This parameter is known to have influence in the bandwidth of the stopband [16]. The smaller $h_{a}$ is the larger the bandwidth obtained. Because of this, if small $h_{a}$ is used, a flatter response is obtained, and thus, less dispersion. Therefore, using a small $h_{a}$ is a suitable solution when reduced dispersion GGW is required. 


\section{B. First row pin modification}

In Fig. 16 the effect of varying width $w_{1}$ of the first row of pins is shown. It is found that the stopband width remains quite stable. For larger $w_{1}$ the increase of capacitance that takes place makes the reactance less negative and compensates the inductances of the structure so that flatter curves are obtained. This kind of modification seems suitable when a less dispersive waveguide is required. On the other hand, it is observed that when pin width is reduced the inductances of the structure grows considerably (see $w_{1}=0,1 \mathrm{~mm}$ ). It has been also checked that increasing pin width only in the $\hat{x}$ direction produces the effect of keeping fixed the stopband and the dispersion curve slope while moving the resonance frequency towards the center of the band.

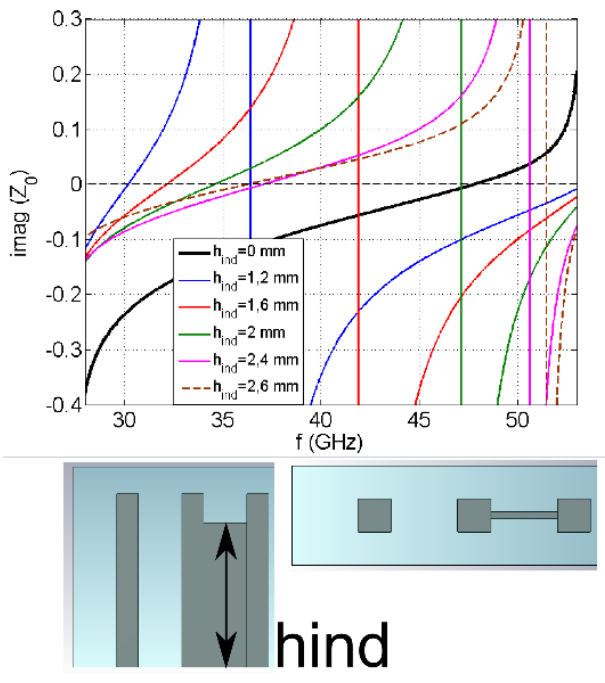

Fig. 17. $Z_{l n}(f)$ vs. $h_{a}$

\section{Introduction of series inductive elements}

The possibility of introducing series inductive elements is now presented. From a practical point of view a simple element with a series inductive behavior could be a thin sheet interconnecting the pins. Fig. 17 shows the result of the parameterization of height $h_{\text {ind }}$ when a sheet is placed between the pins of the first and second row. The width of the sheet has little influence so its width is fixed at $w_{\text {ind }}=60 \mu \mathrm{m}$ Small values of $h_{\text {ind }}$ produce an appreciable narrowing of the stopband, displacement to lower frequencies and highly dispersive behavior. When $h_{\text {ind }}$ reaches the height of the pins $h_{p}$, the slope of the dispersion curve and the stopband bandwidth are similar to the case without insert, however the resonance frequency is more centered and there is a displacement to lower frequencies. A second series inductive element connecting the second and third row $\mathrm{s}$ produces a similar effect. Thus small values of $h_{\text {ind }}$ could be used to design very dispersive GGW while $h_{\text {ind }} \geq h_{p}$ could be used to center the resonance frequency. Furthermore, the observed frequency displacement to lower frequencies for this case could be used to compact the structure.

\section{CONCLUSIONS}

This paper has shown that the direct equivalent correspondence normally assumed between the GGW and rectangular waveguide is a rough approximation which gives accurate results only for specific cases. Also, for the first time, the behaviour of GGW below cutoff has been studied. This study has shown that the GGW and rectangular waveguide behave in a very different manner in terms of their dispersion characteristics. A simple method based on transmission line analysis has been presented which has good predictive capability both below and above cutoff. The model significantly reduces computation time and provides a tool for fast parametric studies for synthesis by repeated optimization. Using the model it is deduced that the GGW is equivalent to a virtual rectangular waveguide whose width grows with the frequency.

\section{ACKNOWLEDGMENT}

This work has been supported by the Spanish Ministry of Science and Innovation under the projects TEC2010-20841C04-01 and CSD2008-00068.

\section{REFERENCES}

[1] D. Lockie and D. Peck, "High-data-rate millimeter-wave radios", Microwave Magazine, IEEE, vol. 10, no. 5, pp. 75-83, 2009.

[2] J. Wells, "Faster than fiber: The future of multi-g/s wireless", Microwave Magazine, IEEE, vol. 10, no. 3, pp. 104-112, 2009.

[3] T. S. Rappaport, S. Sun, R. Mayzus, H. Zhao, Y. Azar, K. Wang, G. N., J. K. Schulz, M. Samimi, and F. Gutierrez, "Millimeter wave mobile communications for $5 \mathrm{G}$ cellular: It will work!” Access, IEEE, vol. 1, pp. 335-349, 2013.

[4] J. Hasch, E. Topak, R. Schnabel, T. Zwick, R. Weigel, and C. Waldschmidt, "Millimeter-wave technology for automotive radar sensors in the $77 \mathrm{GHz}$ frequency band”, Microwave Theory and Techniques, IEEE Transactions on, vol. 60, no. 3, pp. 845-860, 2012.

[5] C. Yeh and F. Shimabukuro, The essence of dielectric waveguides Springer Verlag, 2008.

[6] M. N. Afsar, "Dielectric measurements of millimeter-wave materials", Microwave Theory and Techniques, IEEE Transactions on, vol. 32, no. 12, pp. 1598-1609, 1984.

[7] H. Shigesawa, M. Tsuji, and A. A. Oliner, "Simultaneous propagation of bound and leaky dominant modes on printed-circuit lines: A new general effect”, Microwave Theory and Techniques, IEEE Transactions on, vol. 43, no. 12, pp. 3007-3019, 1995.

[8] F. Mesa, A. Oliner, D. Jackson and M. J. Freire, "The influence of a top cover on the leakage from microstrip line”, Microwave Theory and Techniques, IEEE Transactions on, vol. 48, no. 12, pp. 2240-2248, 2000.

[9] C. Vicente and H. L. Hartnagel, "Passive-intermodulation analysis between rough rectangular waveguides flanges”, Microwave Theory and Techniques, IEEE Transactions on, vol. 53, no. 8, pp. 2515-2525, 2005.

[10]P.-S. Kildal, "Three metamaterial-based gap waveguides between parallel metal plates for mm/submm waves”, in Antennas and Propagation 2009. EuCAP 2009. $3^{\text {rd }}$ European Conference on. IEEE, 2009, pp. 28-32.

[11] A. Valero-Nogueira, M. Baquero, J. I. Herranz, J. Domenech, E. Alfonso, and A. Vila, "Gap waveguides using a suspended strip on a bed of nails", Antennas and Wireless Propagation Letters, IEEE, vol. 10, pp. 1006-1009, 2011.

[12]E. Alfonso, A. Zaman, and P. Kildal, "Ka-band gap waveguide coupledresonator filter for radio link diplexer application”, Components, Packaging and Manufacturing Technology, IEEE Transactions on, vol. 3, no. 5, pp 870-879, 2013.

[13] A. del Olmo-Olmeda, M. Baquero-Escudero, V. E. Boria-Esbert, A. ValeroNogueira, and A. Berenguer, "A novel band-pass filter topology for millimeter-wave applications based on the groove gap waveguide”, in International Microwave Symposium. IEEE, 2013, pp. 1-4.

[14]S. Martínez Giner, A. Valero-Nogueira, J. Herranz Herruzo, and M. Baquero Escudero, "Excitation of untilted narrow-wall slot in Groove gap 
waveguide by using a parasitic dipole”, in Antennas and Propagation (EuCAP), 2013 7th European Conference on, 2013, pp. 3082-3085.

[15]H. Kirino and K. Ogawa, “A 76 GHz multi-layered phased array antenna using a non-metal contact metamaterial waveguide”, Antennas and Propagation, IEEE Transactions on, vol. 60, no. 2, pp. 840-853, 2012.

[16]E. Rajo-Iglesias and P.-S. Kildal, "Numerical studies of bandwidth of parallel-plate cut-off realised by a bed of nails, corrugations and mushroomtype electromagnetic bandgap for use in gap waveguides”, IET microwaves, antennas \& propagation, vol. 5, no. 3, pp. 282-289, 2011.

[17] A. Polemi and S. Maci, "Closed form expressions for the modal dispersion equations and for the characteristic impedance of a metamaterial-based gap waveguide”, Microwaves, Antennas \& Propagation, IET, vol. 4, no. 8, pp. 1073-1080, 2010.

[18] M. Bosiljevac, A. Polemi, S. Maci, and Z. Sipus, "Analytic approach to the analysis of ridge and groove gap waveguides-comparison of two methods”, in Antennas and Propagation (EUCAP), Proceedings of the $5^{\text {th }}$ European Conference on. IEEE, 2011, pp. 1886-1889.

[19] M. Bosiljevac, Z. Sipus, and P.-S. Kildal, “Construction of green’s functions of parallel plates with periodic texture with application to gap waveguidesa plane-wave spectral-domain approach, "Microwaves, Antennas Propagation, IET, vol. 4, no. 11, pp. 1799-1810, 2010.

[20] H. Raza, J. Yang, P.-S Kildal, and E. Alfonso, “ Resemblance between gap waveguides and hollow waveguides”, Microwaves, Antennas Propagation IET, pp. 1-7, 2013.

[21] A. Berenguer, M. Baquero-Escudero, D. Sanchez-Escuderos, and F. Vico, "Rigorous method for calculating gap waveguides impedance using transmission line theory”, in Antennas and Propagation (EuCAP), $20148^{\text {th }}$ European Conference on, 2014, pp. 1-5.

[22] G. F. Craven and C. Mok, "The design of evanescent mode waveguide bandpass filters for a prescribed insertion loss characteristic”, Microwave Theroy and Techniques, IEEE Transactions on, vol. 19, no. 3, pp. 295-308, 1971.

[23] G. F. Craven and R. F Skedd, Evanescent mode microwave components 1987, vol. 1.

[24]P. Ludlow and V. Fusco, "Reconfigurable small-aperture evanescent waveguide antenna”, Antennas and Propagation, IEEE Transactions on, vol. 59, no. 12, pp. 4815-4819, 2011.

[25] P. Ludlow, V. Fusco, G. Goussetis, and D. E. Zelenchuk, “Applying bandpass filter techniques to the design of small-aperture evanescent-mode waveguide antennas”, Antennas and Propagation, IEEE Transactions on, vol. 61, no. 1, pp. 134-142, 2013.

[26] E. Pucci, A. Zaman, E. Rajo-Iglesias, P.-S. Kildal, and A. Kishk, "Study of q.factors of ridge and Groove gap waveguide resonators”, Microwaves, Antennas Propagation, IET, vol. 7, no. 11, pp. 900-908, 2013.

[27] A. U Zaman, P.-S. Kildal, and A. A. Kishk, "Narrow-band microwave filter using high-Q groove gap waveguide resonators with manufacturing flexibility and no sidewalls" Components, Packaging and Manufacturing Technology, IEEE Transactions on, vol. 2, no. 11, pp. 1882-1889, 2012.

[28] C.C. S. Technology, “CST studio suite”, 2013, Suite 2013, Germany.

[29] P.-S. Kildal, A. U .Zaman, E. Rajo-Iglesias, E. Alfonso, and A. ValeroNogueira, "Design and experimental verification of ridge gap waveguide in bed of nails for parallel-plate mode suppression”, Microwaves, Antennas \& Propagation, IET, vol. 5, no. 3, pp. 262-270, 2011.

[30]C. Balanis, Advanced Engineering Electromagnetics. Wiley New York, 1989.

[31] G. Conciauro, M. Bressan, and C. Zuffada, "Waveguide Modes via an integral equation leading to a linear matrix eigenvalue problem”, Microwave Theory and Techniques, IEEE Transactions on, vol. 32, no. 11, pp. 14951504, 1984.

[32] H. Auda and R. F. Harrington, "Inductive posts and diaphragms of arbitrary shape and number in a rectangular waveguide”, Microwave Theory and Techniques, IEEE Transactions on, vol. 32, no. 11, pp. 606-613, 1984.

[33] L. N. Trefethen and D. Bau III, Numerical linear algebra,. Siam, 1997, vol. 50.

[34] M. Abramowitz and I. A. Stegun, Handbook of mathematical functions. Dover publications Inc. New York, 1965.

[35]A. Ishimaru and H.-S. Tuan, "Frequency scanning antennas", in IRE International Convention Record, vol. 9. IEEE, 1961, pp. 101-109.

[36] A. Fackelmeier and E. Biebnl, "Narrowband frequency scanning array antenna at $5.8 \mathrm{GHz}$ for short range imaging”, in Microwave Symposium Digest (MTT), 2010 IEEE MTT-S International. IEEE, 2010, pp. 12661269.
[37]R. M. Foster, “A reactance theorem”, Bell System Technical Journal vol. 3, no. 2, pp. 259-267, 1924.

[38] A. Berenguer, M. Baquero-Escudero, D. Sanchez-Escuderos, and F. Vico, "Reduction of the impedance dependence on the suspended strip gap waveguide”, in USNC/URSI National Science Meeting. IEEE 2014, p. 1.

[39] S. E. Sussman-Fort and R. M Rudish, "Non-foster impedance matching of electrically-small antennas", Antennas and Propagation, IEEE Transactions on, vol. 57, no. 8, pp. 2230-2241, 2009.

[40] S. Maci, M. Caiazzo, A. Cucini, and M. Casaletti, “A pole-zero matching method for EBG surfaces composed of a dipole FSS printed on a grounded dielectric slab", Antennas and Propagation, IEEE Transactions on, vol. 53, no. 1, pp 70-8, 2005.

[41]A. E. Ruehli, "Equivalent circuit models for three-dimensional multiconductor systems" Microwave Theory and Techniques, IEEE Transactions on, vol. 22, no. 3, pp. 216-221, 1974.

[42] J. D. Baena, J. Bonache, F. Martín, R. M. Sillero, F. Falcone, T. Lopetegui, M. A. Laso, J. Garcia-Garcia, I. Gil, M. F. Portillo et al., "Equivalent circuit models for Split-ring resonators and complementary Split-ring resonators coupled to planar transmission lines”, Microwave Theory and Techniques, IEEE Transactions on, vol. 53, no. 4, pp. 1451-1461, 2005. 